\title{
Planning and Implementing Resource Discovery Tools in Academic Libraries
}

Mary Pagliero Popp

Indiana University, USA

Diane Dallis

Indiana University, USA

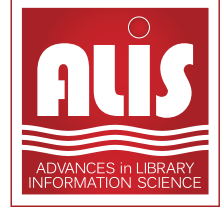


Managing Director:

Senior Editorial Director:

Book Production Manager:

Development Manager:

Development Editor:

Assistant Acquisitions Editor:

Typesetter:

Cover Design:
Lindsay Johnston

Heather A. Probst

Sean Woznicki

Joel Gamon

Hannah Abelbeck

Kayla Wolfe

Milan Vracarich, Jr.

Nick Newcomer

Published in the United States of America by

Information Science Reference (an imprint of IGI Global)

701 E. Chocolate Avenue

Hershey PA 17033

Tel: $717-533-8845$

Fax: 717-533-8661

E-mail: cust@igi-global.com

Web site: http://www.igi-global.com

Copyright (C) 2012 by IGI Global. All rights reserved. No part of this publication may be reproduced, stored or distributed in any form or by any means, electronic or mechanical, including photocopying, without written permission from the publisher. Product or company names used in this set are for identification purposes only. Inclusion of the names of the products or companies does not indicate a claim of ownership by IGI Global of the trademark or registered trademark.

\section{Library of Congress Cataloging-in-Publication Data}

Planning and implementing resource discovery tools in academic libraries / Mary Pagliero Popp and Diane Dallis, editors. pages $\mathrm{cm}$

Summary: "This book addresses the many new resource discovery tools and products in existence as well as their potential uses and applications"--Provided by publisher.

Includes bibliographical references and index.

ISBN 978-1-4666-1821-3 (hardcover) -- ISBN 978-1-4666-1822-0 (ebook) (print) -- ISBN 978-1-4666-1823-7 (print \& perpetual access) (print) 1. Online library catalogs. 2. Federated searching. 3. Library web sites. 4. Library catalogs and users. 5. Information behavior. 6. Academic libraries--United States--Case studies. 7. Academic libraries--Canada--Case studies. I. Popp, Mary Pagliero, 1949- II. Dallis, Diane, 1971-

Z699.35.C38P58 2012

025.5 '24--dc23

2012003667

British Cataloguing in Publication Data

A Cataloguing in Publication record for this book is available from the British Library.

All work contributed to this book is new, previously-unpublished material. The views expressed in this book are those of the authors, but not necessarily of the publisher. 


\title{
Chapter 29 \\ The Long and Winding Road: Implementing Discovery at Indiana University Bloomington Libraries
}

\author{
Courtney Greene \\ Indiana University, USA
}

\begin{abstract}
In early 2010, Indiana University Bloomington Libraries became a beta tester for the EBSCO Discovery Service (EDS) ${ }^{\mathrm{TM}}{ }^{1}$ product, and subsequently selected it in September of that year. After working through various issues with library content and staff expectations, the IUB Libraries launched EDS ${ }^{\mathrm{TM}}$ - branded as OneSearch@IU,in August 2011.This case study provides an overview of the decision-making process and challenges encountered in the process of implementation of a Web-scale discovery tool. Specifictopics such as working with a vendor Application Program Interface (API) to integrate a discovery tool within a library website, formulating an effective extract of library catalog records to import into a discovery tool, customizing a vendor discovery interface, and assessing use and user satisfaction with a discovery system are described. Broad concepts addressed in this chapter include information technology project implementation and library information systems.
\end{abstract}

DOI: $10.4018 / 978-1-4666-1821-3 . c h 029$

Copyright (C) 2012, IGI Global. Copying or distributing in print or electronic forms without written permission of IGI Global is prohibited. 


\section{INTRODUCTION}

As an institution, the Indiana University Libraries are committed to the idea of facilitating discovery, and as a Web-scale discovery tool, EBSCODiscovery Service $(E D S)^{\mathrm{TM}}$ is one part of an overall effort to unify the Library Web presence and present the Libraries' collections, services and resources in a way that enables a more holistic approach to supporting teaching, learning and research in the Indiana University community.

In an article, published in 2006, Dempsey describes a leveraged discovery environment (a vended solution, one outside the library's control) - a concept quite familiar to us now - and cites as its primary goal to bring users back into the local library catalog. For library users, the gap between any library collection that is findable (cataloged) and the portion of the collection that is available or obtainable in a networked environment is "the difference between discovery (identifying resources of interest) and location (identifying where those resources of interest are actually available)." (Dempsey, 2006)

In recent years, the IUB Libraries have prioritized providing broader access to catalog data through new discovery tools with powerful, user-friendly interfaces. In early 2010, Indiana University Bloomington Libraries became a beta tester for EDSTM; it was selected in September of that year and launched in August 2011, branded as OneSearch@IU.A related project in the works is the implementation of an open-source discovery layer (Blacklight) as the primary OPAC interface overlaid on the existing SIRSIDynix ${ }^{\circledR 2}$ ILS system. Scheduled for a summer 2012 launch, the transition to a new public interface will be followed by a complete migration from SIRSIDynix ${ }^{\circledR}$ to the community-sourced Kuali ${ }^{\circledR 3}$ Online Library Environment infrastructure (currently in development) sometime in the following year. (Kuali Foundation, 2010)

While the ability to integrate catalog results in EDS ${ }^{\text {TM }}$ provides one more means to lead users back to IUCAT, the IU Libraries' shared catalog, at this time there is no single tool with an interface that simultaneously meets existing needs relating to discovery to the Libraries' satisfaction. Indiana University Libraries feel strongly that discovery is a key area for investment of library resources; the library catalog and a Web-scale discovery system, such as EBSCO Discovery Service ${ }^{\mathrm{TM}}$, Summon ${ }^{\mathrm{TM}^{4}}$, for example, or discovery interface (such as Blacklight or VuFind), are seen as complementary tools that work together to better meet the needs of the broadly diverse user group undergraduates (primarily novice users), scholars $\&$ faculty (discipline experts who also typically have more expertise in research), graduate students $\&$ interdisciplinary researchers (somewhere in between the other two groups).

As with any new endeavor, there are competing priorities and areas of uncertainty: how to display data that is meaningful and helpful to users in a number of different systems and interfaces; how to balance the needs for attention to detail and to the user experience with the realities of constrained resources \& staff time, while scaling up to deliver data to multiple systems; and, how much can be accomplished through post-processing catalog extracts, and in which areas must local practices be carefully re-examined?

This case study provides an overview of the decision-making process that led to the selection of EBSCO Discovery Service ${ }^{\mathrm{TM}}$ by the IU Bloomington Libraries, and its subsequent implementation, focusing on the integration of EDS ${ }^{\text {TM }}$ into the library Website, and the challenges encountered in working to optimize display and search of catalog data.

When embarking on a project of this kind, goals exist at different levels: first, and most simple to define and later assess, is the goal of successful implementation - that is, to complete the steps necessary to select a product, load the data, customize the interface, then make the product available to end-users. Despite this seemingly straightforward process, numerous unforeseen 
complications and difficulties inevitably arise throughout the project lifespan. In an environment where so much is new, in which the environment (locally and within the marketplace) is constantly evolving, it can be difficult to set long-term goals with much specificity. Indeed, throughout the course of this implementation, changes both to the product itself and to local circumstances have required numerous course corrections. In the end, a series of short-term goals, together with a philosophy that embraces this kind of fluidity and views frequent iteration as success, proved most effective. Throughout, both positive and negative outcomes were valued as providing data leading to better decision-making in future.

The goals for the implementation were also influenced by the vision for change for the library's Web presence, one that conceptualizes not simply a migration and redesign, but a re-envisioning of the methods by which information, services, and resources are seamlessly delivered to users in a variety of venues with a high degree of personalization: directly via the Website, via the campus portal, using mobile devices, on- or off-campus, etc. With an emphasis on flexibility, this vision relies on the ability to reuse and re-mix content to be provided at the time and place of need and provide scaffolding to lead to other resources and services as appropriate. The implementation of EDS $^{\mathrm{TM}}$ is a crucial part of this strategy to deliver library services and resources as effectively and efficiently as possible in as many venues as possible.

\section{BACKGROUND}

The IU Bloomington Libraries serve a population of almost 3000 faculty and over 40,000 students in a wide range of undergraduate, masters, doctoral and post-doctoral programs. Named the top university library by the Association of College and Research Libraries in 2010, the IUB Libraries hold extensive print and electronic collections: well over seven million books, journals, maps, films, and audio/visual materials in over 900 languages; access to more than 600 databases, and many thousands of e-journals, and e-books; and locally developed digital content (Indiana University Bloomington Libraries, 2010). Statewide, Indiana University has eight campuses, with a total enrollment of nearly 110,000 students in fall 2010. (University Institutional Research and Reporting, 2010a) The Indiana University Libraries system includes libraries at each campus location.

Known for its natural and architectural beauty, the Bloomington campus is the largest within the statewide system, both in size (approximately 2000 acres) and in enrollment, accounting for nearly forty percent of total system enrollment. (University Institutional Research and Reporting, 2010b) Founded in 1820, its status as the flagship campus results in a history rich with tradition paired with a commitment to research, teaching and scholarship, which earned it a designation as a Carnegie Research I University. Graduate and undergraduate programs in a variety of disciplines are highly ranked, including the well-known Jacobs School of Music, Kelley School of Business, and Maurer School of Law; and in 2006, IUB placed third on PC Magazine's Top 20 Wired Colleges, the highest ranked public university on that list. (IU News Room, 2011; Jacobowitz, 2006)

The culture of the Bloomington campus balances between a conscious preservation of the traditional and an emphasis on innovation, experimentation and the interest in breaking new ground. These factors are combined with some sense of responsibility to lead, technologically and in other areas, both for the benefit of other campuses in the IU system, and amongst peers nationally and globally. This culture also manifests in the goals and direction of the Libraries, and has influenced decision-making in embracing new approaches, actively contributing to open-source communities such as Kuali ${ }^{\circledR}$, the Sakai Project, and Blacklight, and moving forward to implement technologies as they develop - such as within the emerging discovery marketplace. 


\section{IMPLEMENTING DISCOVERY AT IUB LIBRARIES}

\section{Initial Efforts}

Like scores of other libraries, IU implemented federated search technology branded as OneSearch@IU in 2005.For its many flaws, federated search was an important step forward on the road to discovery, but was not the solution either users or librarians had hoped it might be. (Fagan, 2011) In 2008, IUB Libraries went live with WorldCat Local ${ }^{\circledR 5}$ (WCL); the primary motivator for this decision was user dissatisfaction with the library catalog interface. The dissatisfaction had manifested itself through feedback collected in several ways: focus groups, the LIBQUAL study completed earlier that year, and in regular, annual surveys administered by University Information Technology Services (UITS), the unit overseeing all campus computing services. Some specific examples of desired functionality which $\mathrm{WCL}^{\circledR}$ successfully provided include: search and display of results in non-Roman alphabets (e.g., Cyrillic, Arabic, Hebrew, East Asian languages); improving users' ability to find a known item; allowing the easy creation of lists of items that could then be publicly shared; and achieving better response times on searches. Unfortunately, despite improvements in the user experience overall, some specific needs of the libraries' constituency were still going unmet - namely, since $\mathrm{WCL}^{\circledR}$ does not allow the inclusion of electronic journal, title records from sources such as Serials Solutions ${ }^{\circledR 6}$, nor e-books from aggregators, a significant portion of Library content was inaccessible to users. Further, patrons struggled with the interface, finding it difficult to interpret the way that $\mathrm{WCL}^{\circledR}$ combined records in a version of FRBR.

While in many ways quite successful, $\mathrm{WCL}^{\circledR}$ ultimately proved not to be the right fit for this organization. The previously mentioned issue related to display and search of non-Roman characters was resolved by undertaking a major software upgrade to enable support for Unicode in the SIRSIDynix ${ }^{\circledR}$ catalog in December 2010. Over time, staff support for $\mathrm{WCL}^{\circledR}$ waned, as did its usage, resulting in the eventual termination of the subscription in March 2011.

\section{Enter EBSCO}

In early 2010, EBSCO approached the IU Bloomington Libraries with an invitation to be a beta partner in testing EBSCO Discovery Service ${ }^{\mathrm{TM}}$. Students, faculty and staff responded favorably to the product and to the interface. The timing of this event coincided with a point in the $\mathrm{WCL}^{\circledR}$ project where it had become clear that it would be necessary to reconsider the options available in the market, rather than being able to meet the need for a discovery tool within the context of the evolution of a product (that is, WorldCat Local ${ }^{\circledR}$ ) selected to fulfill a different purpose.

EDS $^{\text {TM }}$ was particularly attractive for several reasons. First, the state of Indiana has an ongoing contract with EBSCO to provide a number of databases that, together with Gale $^{\circledR 7}$ databases, make up INSPIRE, Indiana's Virtual Library, freely available to anyone with an IP address within Indiana. (Indiana State Library, n.d.) This promotes heavy usage of EBSCO resources at every grade level and by the general public, and results in a high level of familiarity with the EBSCOHost interface and recognition for the EBSCO brand with a large proportion of the University constituency. Secondly, EBSCO's implementation process, which was not technically intensive, and their high-touch customer support were especially appealing given the constraints on staff availability. The IU Bloomington Libraries had recently reorganized and created a new department responsible for the Libraries' Website and for related services such as a discovery tool. At that time, the department numbered only two: a search was still underway for a department head to lead the new unit, and all development resources were managed by working in coordination with 
Figure 1. IU Bloomington Libraries home page, http://www.libraries.iub.edu

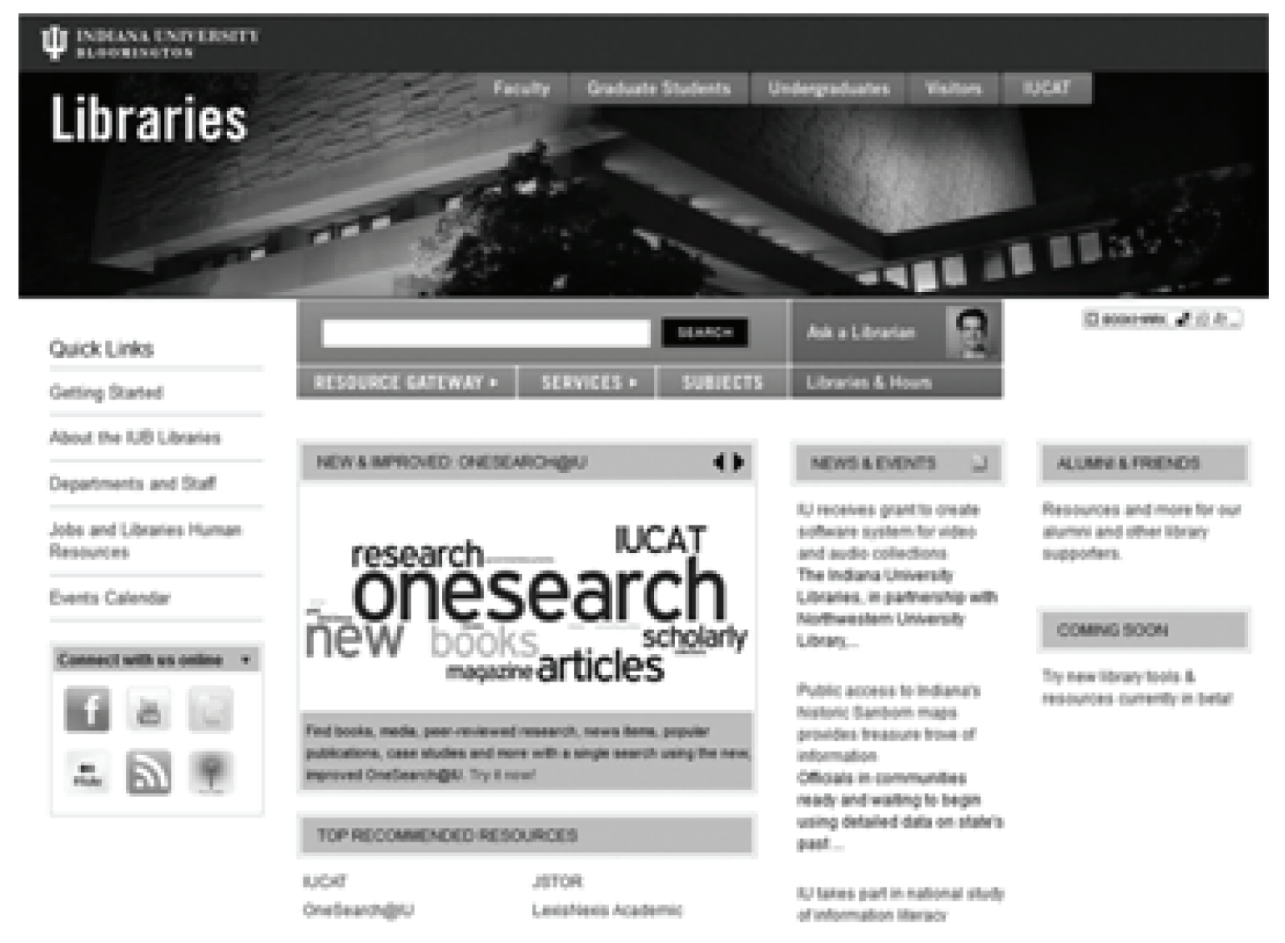

another department outside the library. Finally, in contrast to WorldCat Local ${ }^{\circledR}$, EDS ${ }^{\mathrm{TM}}$ provides direct links to catalog records, giving access to both e-journals and e-books and to local notes for archival and rare book material.

These and other reasons led to the selection of EDS ${ }^{\mathrm{TM}}$ as a Web-scale discovery solution in September 2010. (McEvoy, 2010)

\section{Creating an Integrated Discovery Experience}

As mentioned in the introduction, it has always been a goal to integrate EDSTM into the Libraries' Web presence to the fullest degree possible. Furthermore, setting any loftier goals aside for a moment, a discovery tool is no small investment, and prioritizing this resource contributes toward making the best use of the funds dedicated. The public launch, which took place in August 2011, included the following components:
Branding: Not only are branded services easier to publicize, they are also easier to talk about. The already existing OneSearch@IUbrand,previously associated with a federated search product, was co-opted for use with EDS ${ }^{\mathrm{TM}}$. EDS ${ }^{\mathrm{TM}}$ could then be promoted and publicized at the Bloomington campus as “an all new, improved OneSearch@ IU" - a logical next step in the process of better enabling discovery across all Library collections.

API/Web Service: A major goal of the implementation was to use the EBSCOHost Integration Toolkit (EIT) Web service, or API, to present results from EDSTM within the Library's Website search results. (EBSCOHost, n.d.) It also provided a way to address requests from the student body to integrate more visual cues into search results - format icons, book covers, etc.

The Libraries Website presents users with a single search box (sometimes called the "resource discovery search," or in other cases more simply "the orange box") that retrieves results from a 
Figure 2.IUB Libraries site search results, showing OneSearch@IU results provided via EIT Web service

\section{Search Results}

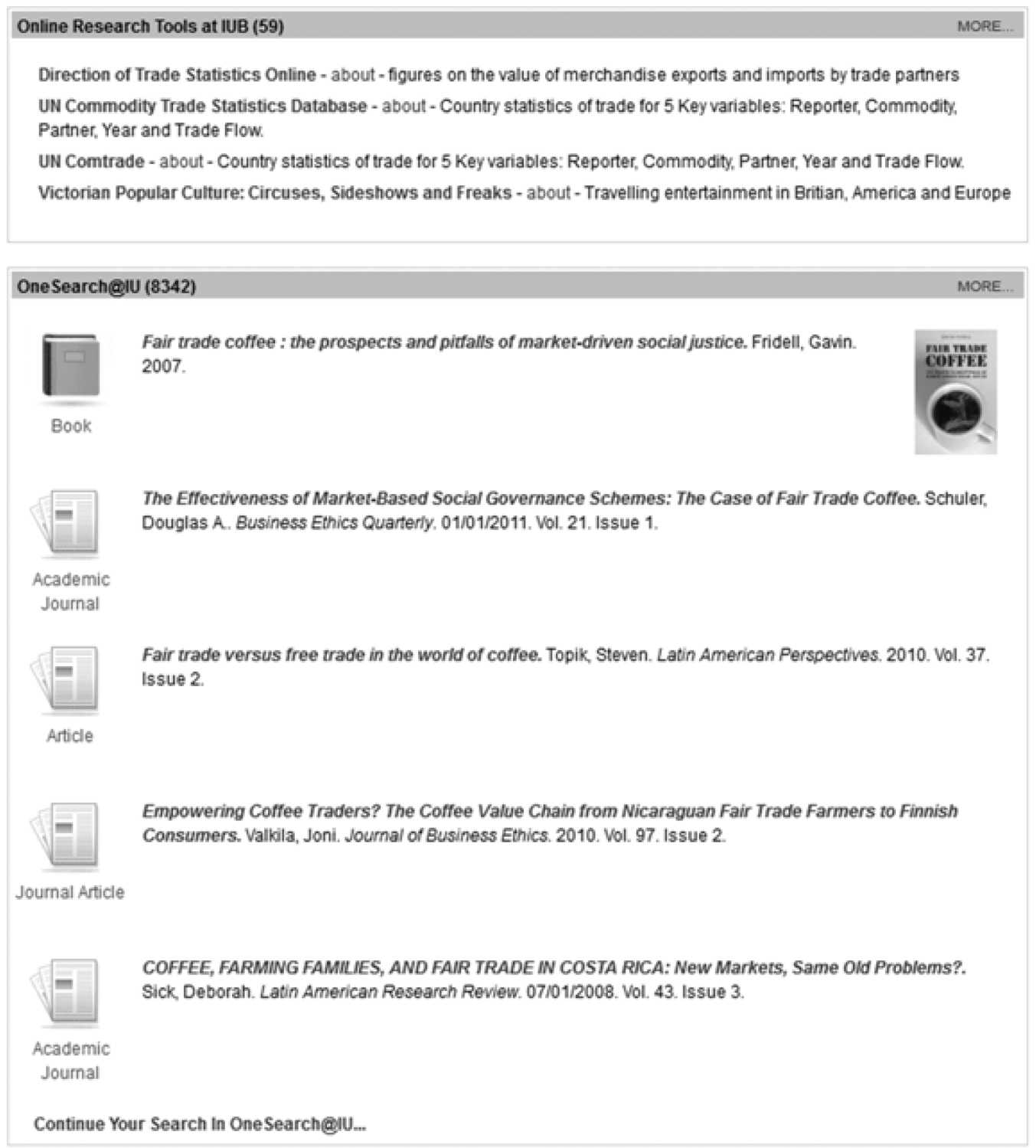

variety of sources, including resource information (databases), Serials Solutions ${ }^{\circledR}$ e-journal list, and Website search. [Figure 1] These results from diverse sources are then assembled on a single page. Previously, results from the federated search product were also returned; at launch, the page was updated to instead display results from EDSTM provided via the EIT Web service. [Figure 2]

Subject pages The EDSTM search box was made available within the Libraries' research guides as a separate tab, in lieu of the federated search option that had previously appeared there. (Figure 3) The intention was to provide easy ac- 
Figure 3. African Studies subject research guide, showing OneSearch@IU tab

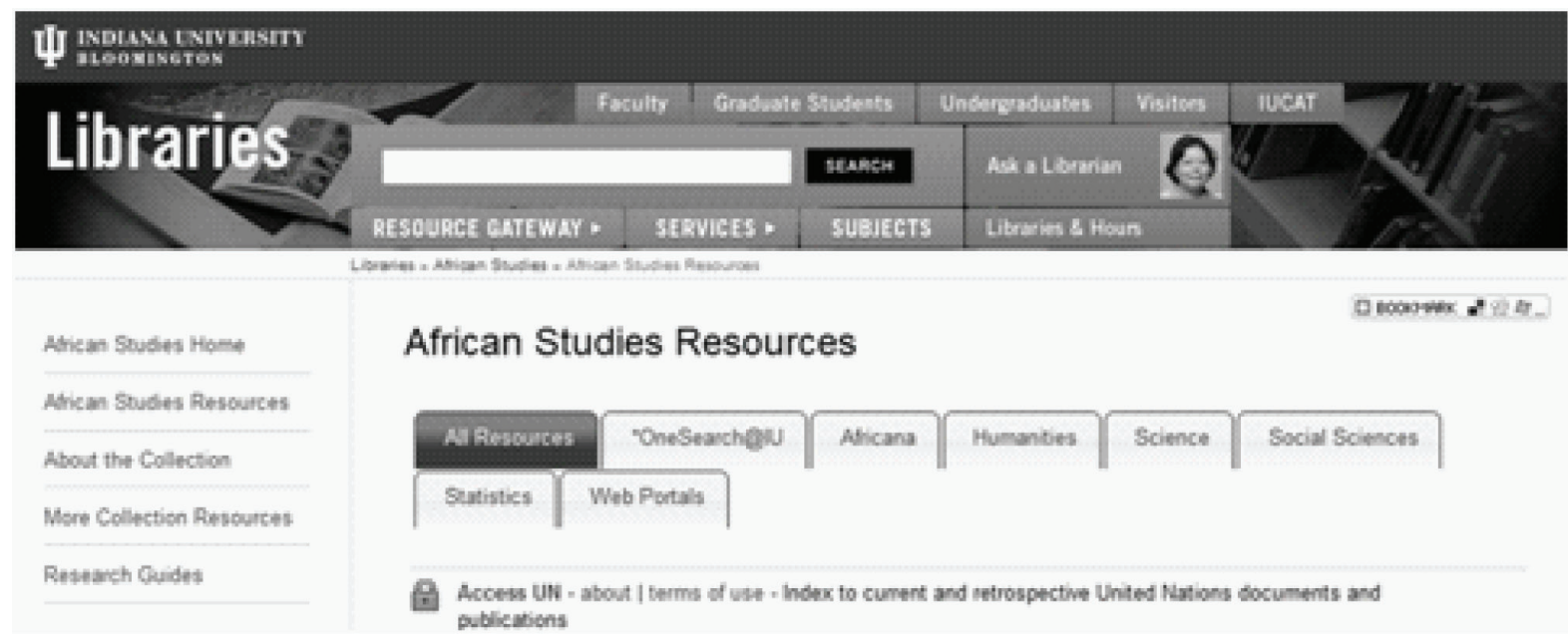

cess to this resource for all users while retaining the carefully constructed resource listings maintained by collection managers. This is viewed as an intermediate step, and with the intention of exploring more active integration in this area.

"Find Information" page The "Find Information" page has historically served as the page to which databases refer users upon ending or exiting a session. While many vendors have moved away from this behavior, the page still sees reasonably heavy use: it indexes high in Google search results for IUB Libraries, and is also the target of a library link appearing within the course management system for a small number of courses not associated with specific departments or programs. This page provided an opportunity to begin testing the integration of search behaviors, such as tabbed search boxes, planned to be integrated throughout the Website in future. (Figure 4)

\section{Implementation: A Moving Target}

Like other discovery solutions available on the market, EDSTM includes information from multiple sources pulled together into a larger data set that is then indexed as a whole: the base index, content from subscribed databases (EBSCO and others), and the catalog extract. The base index, common to all EDS ${ }^{\text {TM }}$ customers, includes citation and abstract data from approximately 20,000 providers (at the time of writing). (EBSCO Publishing, 2011) To this is added metadata from other sources to which the customer subscribes, both EBSCO databases (enabling full-text linking) and other providers with whom EBSCO has negotiated agreements (enabling direct linking to full text in those sources, called "custom links"). Finally, each customer provides an extract of their catalog data - a snapshot, if you will, of the bibliographic records and holdings informationwhich is also included in that customer's EDSTM dataset. EBSCO then indexes all this data together and results are returned based on their proprietary relevancy ranking algorithm.

Some adjustments can be made to the relevancy ranking by the vendor. For example, early in the IUB beta period, results from a specific content source were drowning out results from nearly all other sources, including the catalog, and EBSCO was able to make adjustments to correct this.

Customers cannot alter the contents of the base index, but specific subscribed databases can be included or removed from EDSTM content from 
Figure 4. "Find information" page tabbed search box

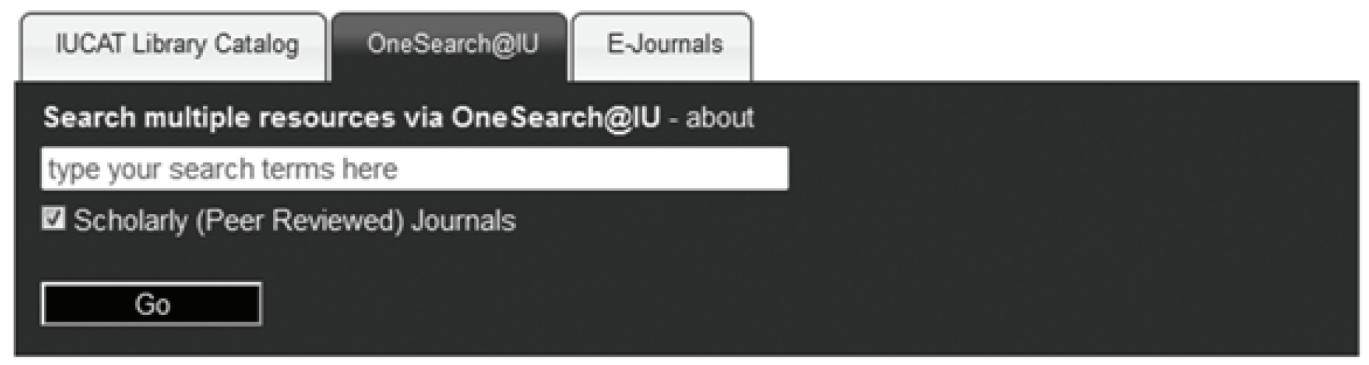

within the administrative interface. The IUB Libraries opted to remove several sources in this way, either because the content was felt to be unnecessary within the specific context of EDS ${ }^{\mathrm{TM}}$ (e.g., Funk and Wagnall's New World Encyclopedia), or the content was useful or usable only to a very narrow set of users (e.g., KISS: Korean Studies International, from which results display in Korean). In one case, content from OAIster (The Open Archives Initiative metadata harvesting protocol) was added and then removed, as results proved to be duplicative with high levels of variance in metadata quality.

\section{Integrated Search}

EDSTM includes "Integrated Search" as a means of including content from data not currently available either through the base index or as additional subscription-based sources. This federated search functionality can be enabled or disabled. Despite reservations about federated search generally, IU Bloomington opted to include a small number of sources (primarily ProQuest ${ }^{\circledR}$ products) at launch. User testing will be conducted in early 2012 to assess its efficacy and the importance of continuing with this feature.

\section{API}

Through the use of the EIT Web service, results from EDS $^{\mathrm{TM}}$ are returned within the search results page in the library Website. In addition to basic citation information (author, title, date), icons indicating item type, and when available, book covers, are presented as part of the results.

Upon querying the EDS ${ }^{\mathrm{TM}}$ partners listserv, an active community for discussion and support, regarding issues relating to the API, it seemed that the primary interest of that group at that time was in integrating search-box code provided by the vendor rather than in working with the API. Happily, Hassan Sheikh, Head of Systems Development for The Open University in the United Kingdom responded with information about similar integrations within their Website, and he was extremely helpful in answering questions. (Sheikh, personal communication, June 17, 2011) The most pressing of the issues encountered was slow response times for results which initially might take as long as twenty seconds to return. EBSCO also proved very responsive and made changes which resulted in a huge improvement in result times, with results in just two or three seconds.

Staff reactions to the integration of EDSTM API results on the search results data have been varied, from the very positive to the extremely negative. In some cases it has been unclear whether the dissatisfaction felt centers specifically on the API data, or whether it is more generally related to the overall design of the search results page. To date, no negative comments on the change have been received from users, however; extensive user testing focusing on the search results page 
and specifically on the API results is planned in the near future.

\section{Catalog Extract}

Catalog data is extracted from the SIRSIDynix ${ }^{\circledR}$ integrated library system and provided to EBSCO in a machine-readable file, which includes the bibliographic record and holdings data for each item. At IUB, updates are provided and loaded weekly. As previously noted, IUCAT is a shared online catalog for the entire Indiana University system, and IU Kokomo, which implemented EDS $^{\text {TM }}$ in summer 2010, shares this same catalog extract. While there has been some discussion of the possibilities of generating multiple extracts and thus enabling individual campuses to load only their holdings, there are no plans to proceed in that manner at this time.

Once the catalog extract is uploaded as part of the EDSTM dataset, EBSCO provides real-time availability checking of location, call number, and status (including due date, if appropriate) for items by communicating with the institution's Z39.50 server. If the Z39.50 server cannot be reached to display the most current data, the holdings data from the extract will be displayed.

Often, it has been in working with the catalog extract that the most interesting challenges have arisen, and these have accounted for the bulk of the time elapsed in the period spanning from adoption to public launch.

A great deal of time has been invested in customizations to the interface of the SIRSIDynix ${ }^{\circledR}$ OPAC, smoothing out rough places, introducing improvements where possible, and generally tidying up. After such careful consideration of how the catalog data and the interface of the ILS work together-making countless adjustments, both to highlight and to obscure various characteristics of the system (SIRSIDynix's ${ }^{\circledR}$ double tab) or pieces of information (system-generated call numbers) - viewing the data in a new interface was a real eye-opener.
Over seven million records representing a wide array of formats provided plenty of opportunity for detecting anomalies, or sub-optimum data display (that is to say, records that looked just plain ugly, wherever they were viewed). Testing identified a few classes of records that were duplicative, unhelpful, or problematic (for example, stub records provided by vendors). Early in the process, the decision was made to remove title-level electronic journal records provided by Serials Solutions ${ }^{\circledR}$ from the extract with the rationale that these records did not add value for users within the context of searching EDSTM. Ebook records obtained from Serials Solutions ${ }^{\circledR}$ have been retained since it is judged that these do increase access for users.

In some cases, records from specific locations have been filtered. Many libraries in the IU system share records in IUCAT. Other libraries do not, for specific reasons: The Lilly Library and Music Library each have their own records because they add extra information to the record that is appropriate for their specialized collections; and the professional health schools (Medicine and Dentistry) share a record. In other cases, separate records are created for specific collections or locations: the Archives of Traditional Music, Kinsey Institute Library, Bloomington Law Library, and the Bloomington Residential Programs and Services Libraries are extra-systems libraries and their materials are not owned by the IU Bloomington Libraries. Specifically, in Kinsey records, additional data such as donor information is often added; because those items do not circulate, and because that private donor information is part of a notes field that cannot be suppressed from display for all records, Kinsey records have been filtered out of the catalog extract provided to EBSCO.

As these and other classes of records have been filtered out of the extract, new sources of clutter have been revealed. Over the long term, as the venues in which IUCAT data can be discoverable are further expanded, local cataloging practices 
will need to be carefully considered to enable consistency across discovery interfaces.

EBSCO enables customization of display of catalog records both within the result list and for the detailed bibliographic record. Significant improvements were achieved by working with them to suppress certain fields or specific data. For example, fields that were suppressed following launch were the 050 (call number; this often differs from the local call number, stored in the 999), the 024 (other standard number, such as UPC if included), and the 074 (Government Printing Office item number) field. It is also possible to suppress only certain data from a given field system-generated call numbers, for example. All of this data is available in the IUCAT full display, easily accessible in just one click; within EDS ${ }^{\mathrm{TM}}$, this information was confusing to users and so it was eliminated.

In many cases, records downloaded from OCLC $^{\circledR}$ include additional information that is not useful locally. While in some cases the record is edited prior to inclusion in the local database, in some cases data is simply suppressed from display in the OPAC - foreign language subject headings, as one example. However, because this data remained in the record, it was visible within OneSearch@IU, so that SteveKrug's bookRocket Surgery Made Easy showed subjectheadings of not just "Web-based userinterfaces - Evaluation," but also for "Benutzeroberfläche," the German heading. This was corrected by requesting thatEBSCO suppress all values of $65 \mathrm{x}$ (subject heading) fields with indicator 7 (denoting foreign language).

In some cases, where multiple records existed for the same item (for the reasons mentioned above), the holdings would simply ... "jump."An example-there are two records for The Catcher in the Rye: copy 1 shows holdings for IUB, IUPUI, and IU East; copy 2 shows a single holding for Residential Programs and Services. Sometimes, copy 1 would show all 4 holdings records while copy 2 showed none; other times, it might be the reverse. (Figure 1,2) It was this very changeable- ness that slowed resolution, because results could not always be reliably replicated.

After a period of extensive testing, it was determined that the problem arose from multiple records with the same value in the 001 field. The 001 field is generally used as a unique identifier for records; because in these cases, multiple records were assigned the same value in the 001 , there was no way to use the 001 to re-assemble them properly, or consistently, when loaded into OneSearch@IU. In SIRSIDynix ${ }^{\circledR}$, the systemassigned unique record ID number is the "ckey" (catalog key) number. This was already being used within EDS ${ }^{\mathrm{TM}}$ to point to the appropriate record in IUCAT, both from the search results list and the full bibliographic record. When viewing a catalog record within EDS ${ }^{\mathrm{TM}}$, the "retrieve catalog item" link from the search results list or the record itself links with the catalog key to the specific record in IUCAT. (For example, the OneSearch@IU record Rocket Surgery Made Easy [Figure 5] takes the user to the IUCAT record as ' 1 of 1 for search" 9536208 \{ckey\}."'[Figure 6]) The solution, then, was to provide EBSCO with a catalog extract in which the ckey values appeared in the 001 field.

\section{Assessment: User Feedback and Evaluation}

In early 2010, a representative from EBSCO Publishing conducted on-campus testing of a beta version of EDS ${ }^{\mathrm{TM}}$ at multiple locations nationwide, including the Herman B Wells Library on the Bloomington campus. At IUB, a total of seven participants (4 undergraduates, 2 graduate students, and one library staff member) each completed at least two scenario-based tasks using the beta version of EDSTM, and each was also asked to test upcoming features using a prototype. Generally, participants reacted favorably, specifically mentioning that EDS ${ }^{\text {TM }}$ was easy to use and that they were pleased by the ability to search a large proportion of the Libraries' content through a single search box. Undergraduates rated their 
The Long and Winding Road

Figure 5. OneSearch@IU record, Rocket Surgery Made Easy

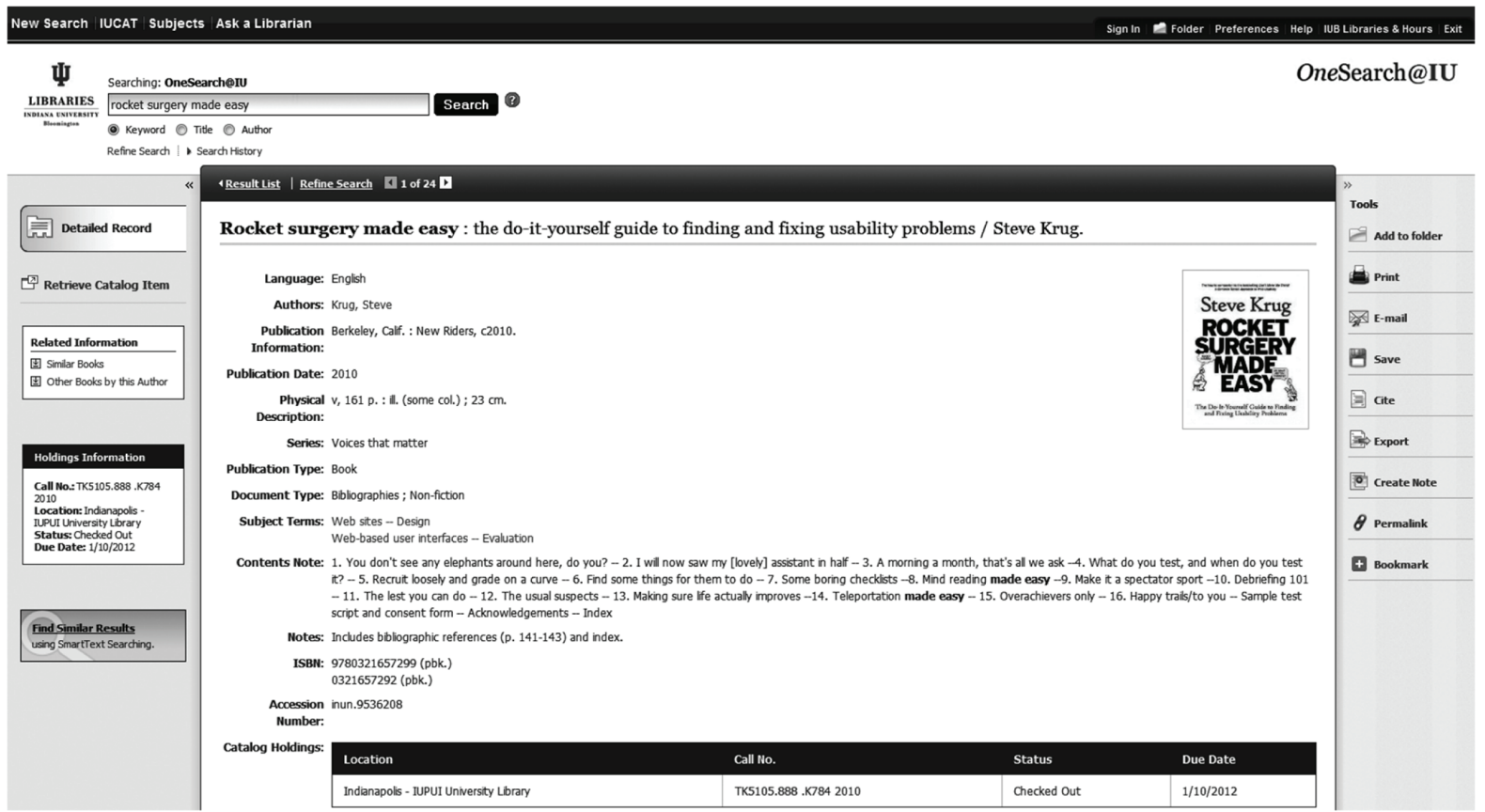

overall experience in using EDS ${ }^{\mathrm{TM}}$ as a 4.25 (on a scale of 1 to 5, with five being 'very usable'). Interestingly, when asked how likely they might be to use EDS ${ }^{\mathrm{TM}}$ again, graduate students' rating was highest - a 5 (on a scale of 1 to 5,5 being 'very likely'), with undergraduates' rating being a 4.25. These positive results were influential in IUB Libraries' decision to subscribe to EDS ${ }^{\mathrm{TM}}$.

OneSearch@IU launched in lateAugust 2011, immediately previous to the start of the fall semester. Marketing and promotion was limited to a feature on the home page of the library Website, fliers (distributed to all campus libraries including those located in the Halls of Residence) and a display in the main lobby of the Herman B Wells Library. Usage has climbed throughout the fall semester, although it is still considerably less than combined usage of all other EBSCO databases. It is hoped that continued promotion in a number of venues, including demonstrating the resource in library instruction sessions, will result in a continued increase in usage.
Internally, amongst library staff, reactions to EDS $^{\text {TM }}$ have been mixed. Initially there was considerable concern of a general nature: what impact a discovery tool might have on user behavior, and whether usage of more specialized databases might be negatively affected; whether a discovery tool adequately served all populations, specifically advanced researchers; and whether it might be incorrectly inferred by users that all library content was available through the discovery tool. While these are certainly worthy questions, they relate more to the overall concept of discovery itself, rather than to any specific tool such as EDSTM, and as such are common to any implementation. In early stages of the beta release, some of the data and display issues detailed in the previous section were also causes of concern to staff. As those have been resolved, most staff have become more positive about OneSearch@IU, with the Reference and Teaching \& Learning departments showing support throughout the implementation process. One specific area in which OneSearch@ IU has been useful in providing reference service 


\section{IUCAT Home | My Account | Interlibrary Loan/Document Delivery/Recall | Ask | Login}

Go Back | Help | New Search | Change Display | Print/Email/Save | Permalink

\begin{tabular}{|c|c|c|}
\hline \multirow{13}{*}{ Keep to print / email / save } & \multicolumn{2}{|r|}{ Details } \\
\hline & \multicolumn{2}{|c|}{$\begin{array}{l}\text { Rocket surgery made easy : the do-it-yourself guide to finding and fixing usability problems } \\
\text { Krug, Steve. }\end{array}$} \\
\hline & $\begin{array}{l}\text { Author: } \\
\text { Title: }\end{array}$ & $\begin{array}{l}\text { Krug, Steve. } \\
\text { Rocket surgery made easy : the do-it-yourself guide } \\
\text { to finding and fixing usability problems / Steve Krug. }\end{array}$ \\
\hline & Published: & Berkeley, Calif. : New Riders, c2010. \\
\hline & Description: & v, 161 p. : ill. (some col.); $23 \mathrm{~cm}$. \\
\hline & Series: & Voices that matter \\
\hline & $\begin{array}{l}\text { Uniform series: } \\
\text { Notes: }\end{array}$ & $\begin{array}{l}\text { Voices that matter. } \\
\text { Includes bibliographic references (p. 141-143) and } \\
\text { index. }\end{array}$ \\
\hline & Contents: & 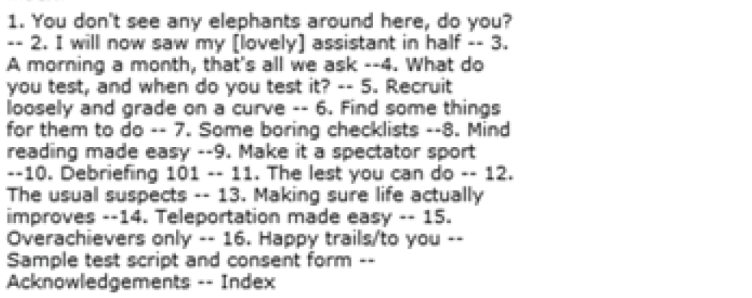 \\
\hline & Summary: & $\begin{array}{l}\text { In this how-to companion to Don't Make Me Think: A } \\
\text { Common Sense Approach to Web Usability, Steve } \\
\text { Krug spells out an approach to usability testing that } \\
\text { anyone can easily apply to their own web site, } \\
\text { application, or other product. --from publisher } \\
\text { description }\end{array}$ \\
\hline & Subject headings: & Web sites-Design. \\
\hline & Subject headings: & Web-based user interfaces--Evaluation. \\
\hline & ISBN: & 9730321657299 (pbk.) \\
\hline & ISBN: & $0321657292(p \mathrm{bk})$. \\
\hline
\end{tabular}

is the ease of providing patrons with permalinks to results sets that include both journal literature and items from IUCAT, the shared catalog - particularly helpful within instant message reference transactions. Undoubtedly, however, the most enthusiastic internal adopters of OneSearch@ IU have been the reference assistants - graduate students from the Indiana University School of Library and Information Science who staff the reference desks at the Wells Library. That group has also been quite helpful in troubleshooting and reporting problem records.

In July 2011, Sarah Williams and Anita Foster published the results of a usability study of the Illinois State University implementation of EDS ${ }^{\mathrm{TM}}$, called SearchIt; the first round of usability testing of OneSearch@IU, planned for early 2012, will build off of their work, using many of the same tasks. (Williams \& Foster, 2011) This initial testing will focus on what changes or improvements can be made to the local configuration options available within EDSTM itself.

Within the EDS ${ }^{\text {TM }}$ partner community, EBSCO has announced plans to continue development of the API/EIT Web Service, and IUB Libraries will participate as a beta tester in that project. Given that the integration of API results into the Library Website has been an important part of the implementation project, and that there is interest in identifying additional for integration of EDS ${ }^{\mathrm{TM}}$ results within the Web presence, the second phase of user testing will focus on the Libraries' search 
results page and the presentation of $\mathrm{EDS}^{\mathrm{TM}}$ results via the API.

\section{FUTURE RESEARCH DIRECTIONS}

Additional areas of interest for future user testing include more in-depth research into use of facets; gauging user awareness of, and perceived value for, the integrated search (federated search) functionality; testing of the mobile application slated to be released by EBSCO sometime in 2012; and, of course, ongoing testing as EBSCO continues to release changes or add features to the product.

On a larger scale, further investigation is needed to begin to assess the impact of discovery tools on user behavior, in general and within specific populations (undergraduates, graduates, faculty, researchers, and for each of those groups within different disciplines). What impact does a discovery tool have on the usage of other databases and of the library catalog? Will discovery tools have the hoped-for impact - that is, increased usage of library resources?

\section{CONCLUSION}

Following the user testing planned for early 2012, several areas of concentration in the medium-term include pursuing more in-depth user feedback through focus groups to investigate how OneSearch@IU might be best marketed to various audiences, and which improvements should be prioritized; and investigating opportunities for campus-level integration of OneSearch@ IU into venues such as OnCourse, the Indiana University course management system (part of the Sakai Project). Efforts are now in progress at other institutions (the University of Michigan [US] and the University of Oxford [UK]) to enable the integration of discovery products from Serials Solutions ${ }^{\circledR}$ and ExLibris ${ }^{\text {TM8 }}$ into Sakai, and there is local interest in doing so at IUB with EDS $^{\mathrm{TM}}$ in 2012.

Looking to the future, however, there are larger questions that must be considered: What is the role of a research library as related to discovery? While the capacity for discovery, such as that delivered by a Web-scale discovery tool, does not increase the size of the information universe. It increases the proportion discoverable to any individual patron; conversely, this sense of expanded capacity further isolates those items and collections not included in the discovery environment. The primary target audience for many discovery solutions - the undergraduate - will feel no lack, either in quantity of results, or in the quality of the user experience through which they are accessed. This is not to say that discovery holds nothing for the graduate student, the faculty member, and the scholar, but simply that those groups tend to have already identified at least a reasonable proportion of the information universe that is likely to be relevant to their research. Discovery in its present incarnation both helps and hinders research libraries in the Libraries' role as information curators, which up to this time has consisted primarily of carefully and thoughtfully selecting, compiling and preserving materials, and of presenting various content silos, both internally and externally created, to their best advantage - which is as they intersect with the needs of the user population. It helps in that silos are reduced, and content is more widely accessible; and it hinders in that now more than ever, what is not explicitly included is implicitly obscured. Doubtless as Web-scale discovery platforms evolve, improvements will be achieved that will mitigate some of the concerns about completeness.

Lorcan Dempsey's assertion that discovery systems exist as a means of directing users back to the library catalog is arguable, not in terms of what it intends to achieve (ultimately, broader usage of library collections), but in terms of how it may best be achieved. Is the user best served by remaining within the local catalog, or can 
better success be achieved by focusing more on integrating catalog data within other systems, such as OneSearch@IU? In 2007, prior to the advent of Web-scale discovery products, Marshall Breeding stated that that any next-generation catalog (NGC) that did not include information from article databases stood incomplete as a service to users. (Breeding, 2007, p. 35) Andrew Nagy in his 2011 Library Technology Reports issue "Analyzing the Next-Generation Catalog," states, "The NGC is a stepping-stone technology, preparing libraries for the migration to Web-scale services ... [it] does not meet user expectations: ... it does not search everything." (Nagy, 2011,pp. 11,26) At this stage, neither the NGC nor the Web-scale discovery platforms can claim to contain everything. Most importantly, even if at some point they should, enabling the fullest degree of discovery for users relies entirely on the quality, completeness, and consistency of Library data, whether the source is the library catalog or a licensed index.

Locally, the parallel efforts both to implement EBSCO Discovery Service ${ }^{\text {TM }}$ and to adopt a new discovery interface for IUCAT, followed by the planned migration to the Kuali ${ }^{\circledR}$ OLE system, underscore the belief that at this time, a Webscale discovery service and a next-generation library catalog are complementary tools that work together to better meet the needs of diverse user communities.

\section{REFERENCES}

Breeding, M. (2007). The birth of a new generation of library interfaces. Computers in Libraries, 27(9), 34-37.

Dempsey, L. (2006). The library catalogue in the new discovery environment: Some thoughts. Retrieved from http://www.ariadne.ac.uk/issue48/ dempsey/

EBSCO Publishing. (2010). EDS usability test report. Unpublished report.
EBSCO Publishing. (2011). EDS content. Retrieved December 28, 2011, from http://www. ebscohost.com/discovery/eds-content

EBSCOHost. (n.d.). EBSCOhost integration toolkit. Retrieved July 31, 2011, from http://eit. ebscohost.com/

Fagan, J.C. (2011). Federated search is dead-And good riddance! Journal of Web Librarianship, 5(2), 77-79. doi:10.1080/19322909.2011.573533

Indiana State Library. (n.d.). Frequently asked questions. INSPIRE Indiana's Virtual Library. Retrieved July 31, 2011, from http://www.in.gov/ library/inspire/faq.html

Indiana University Bloomington Libraries. (2010). IUB libraries: Fact sheet. Retrieved July 31, 2011, from http://www.libraries.iub.edu/index. php?page $\mathrm{Id}=5430$

IU News Room. (2011, March 15). "U.S. News" gives high marks to IU programs in nursing, law, business, education, medicine. Retrieved from http://newsinfo.iu.edu/news/page/normal/17756. html

Jacobowitz, P. (2006, December 20). \#3 Indiana University Bloomington - Top 20 wired colleges. PCMag.com. Retrieved from http://www.pcmag. com/article2/0,2817,2073461,00.asp

Kuali Foundation. (2010). Kuali OLE. Kuali Foundation. Retrieved July 31, 2011, from http:// www.kuali.org/ole

McEvoy, K. (2010, June 7). Indiana University selects EBSCO Discovery Service ${ }^{\mathrm{TM}}$ to give users what they expect online. EBSCO Publishing. Retrieved from http://www.ebscohost.com/discovery/view-news/indiana-university-selects-EDS

Nagy, A. (2011). Analyzing the next-generation catalog. Library Technology Reports, 47(7), 1-27. 
University Institutional Research and Reporting. (2010a). IU fact book 2010: Bloomington. Retrieved from http://www.iu.edu/ uirr/reports/ standard/factbook/campus_fb/2010-11/Bloomington/FactBook1011_BL.pdf

University Institutional Research and Reporting. (2010b). IU fact book 2010-11: University. Retrieved from http://www.iu.edu/ uirr/reports/ standard/factbook/campus_fb/2010-11/University/FactBook1011_Web.pdf

Williams, S., \& Foster, A. (2011). Promise fulfilled? An EBSCO Discovery Service usability study. Journal of Web Librarianship, 5(3), 179-198. doi:10.1080/19322909.2011.597590

\section{ADDITIONAL READING}

Briceño-Rosales, Z. C. (2011). OCLC WorldCat: Interactivity and mobility create a winning combo. Library Journal, 136(Reference Supplement), 15-16.

Coyle, K. (2010). FRBR, the domain model. RDA vocabularies for a twenty-first-century data environment [Special issue]. Library Technology Reports, 46(2), 20.

Fernandez, R. (2011). Ex Libris's Primo: Easy to implement, intuitive to use. Library Journal, 136(Reference Supplement), 17.

Hanson, C., Hessel, H., Barneson, J., Boudewyns, D., Fransen, J., \& Friedman-Shedlov, L. ... Traill, S. (2009). Discoverability phase 1 final report. University of Minnesota Libraries. Retrieved from http://purl.umn.edu/48258

Hanson, C., Hessel, H., Barneson, J., Boudewyns, D., Fransen, J., \& Friedman-Shedlov, L. ... West, A. (2011). Discoverability phase 2 final report. University of Minnesota Libraries. Retrieved from http://purl.umn.edu/99734
Luther, J., \& Kelly, M. C. (2011). The next generation of discovery. Library Journal, 136(5), 66-71.

Newcomer, N. L. (2011). The detail behind Webscale: Selecting and configuring Web-scale discovery tools to meet music information retrieval needs. Music Reference Services Quarterly, 14(3), 131-145. doi:10.1080/10588167.2011.596098

Powers, A. C. (2011). EBSCO's EDS: Relying on patron data to show the way. Library Journal, 136(Reference Supplement), 14-15.

Thornton-Verma, H. (2011). Discovering what works: Librarians compare discovery interface experiences. Library Journal, 136(Reference Supplement), 14.

Varnum, K. (2011). Serials Solutions' Summon: Familiarity breeds success. Library Journal, 136(Reference Supplement), 18.

Vaughan, J. (2011). Web scale discovery services. Library Technology Reports, 47(1), 5-29.

\section{KEY TERMS AND DEFINITIONS}

API (Application Programming Interface): Sometimes used interchangeably with "Web service," an API provides a way for applications to communicate and share data. In other words, by using certain protocols or standards, data can be retrieved, processed, and displayed by other applications and in other contexts than the originating data source. Examples of well-known APIs are the Google Maps API, or the WorldCat ${ }^{\circledR}$ API. One example of the use of an API would be many popular third party Twitter applications (e.g. TweetDeck); these applications query the Twitter API to bring back the data within their own interface.

Community Source: Community source refers to a specific type of open source project in that a specific community entity, often comprised of representatives from supporting institutions or 
organizations, has committed ongoing funding, support and other resources to the project. Examples of well-known community source entities are the Sakai Project (http://sakaiproject.org/) and the Kuali Foundation (http://kuali.org/).

Discovery Tool: A platform featuring a unified index created by bringing together data from a wide array of publishers, vendors and other sources (including library catalog records), resulting in improved relevancy ranking across the entire set. This allows users to simultaneously broaden the scope of their search and to increase the precision of the results returned, and the discovery tool typically presents an attractive interface designed to meet user expectations for ease-of-use, sharing, and other functions common to commercial Websites such as Amazon or Google.

Federated Search: Sometimes referred to as "metasearch" tools, federated search tools allow a single query to be simultaneously delivered to multiple information resources, and then to collect those results and display them as a single set. To accomplish this, the tool must generally rely on "translators" which enable communication with the varied sources.

FRBR(Functional Requirements for Bibliographic Records): For more detailed information, see http://www.ifla.org/publications/functionalrequirements-for-bibliographic-records.

Kuali Online Library Environment: Acommunity sourced initiative to develop and release a product that meets the needs of academic and research libraries to acquire, describe, circulate, and provide access to their collections.
Unicode: A standard that allows for the consistent searching, rendering and display of text across the majority of the world's written languages.

Web Service: A Web service is more or less an API that uses certain Web protocols to communicate. A concrete example of this might be a Web service that maintains information about library hours; information about hours is loaded into a database, which can be communicated with via the Web service; that Web service could then be queried by code placed in a variety of places on the library Website, and this would result in dynamic display of the hours information, always direct from the authoritative source (the database). A helpful analogy for the less technical might be an RSS feed: by subscribing to that RSS feed, its information is delivered to you - the underlying concept of data sharing is the key idea.

\section{ENDNOTES}

1 EBSCO Discovery Service is owned by EBSCO Publishing Industries

2 SIRSIDynix is a registered trademark of SIRSIDynix Corporation.

3 Kuali is an open source administrative software.

4 Summons is owned by ProQuest LLC

$5 \quad$ Worldcat is a registered trademark of OCLC.

6 Serials Solutions is a registered trademark of Serials Solutions

7 Gale is a registered trademark Gof Cengage Learning

8 ExLibris is a trademark of Ex Libris Ltd. 